\title{
Resveratrol upregulates SOCS1 production by lipopolysaccharide- stimulated RAW264.7 macrophages by inhibiting miR-155
}

\author{
CHUNFANG MA, YIN WANG, AIJUAN SHEN and WANRU CAI \\ The Second Affiliated Hospital of Zhejiang Chinese Medical University, Hangzhou, Zhejiang 310005, P.R. China
}

Received December 13, 2015; Accepted November 1, 2016

DOI: $10.3892 / \mathrm{ijmm} .2016 .2802$

\begin{abstract}
Resveratrol is a polyphenolic compound extracted from grapes and the Chinese herb, Polygonum cuspidatum. In the present study, in order to elucidate the molecular mechanisms of action of resveratrol in host immune cells, we examined the effects of resveratrol on the inflammatory response in lipopolysaccharide (LPS)-stimulated RAW264.7 murine macrophages. The cells were treated with resveratrol prior to stimulation with LPS $(1 \mu \mathrm{g} / \mathrm{ml})$. Resveratrol downregulated the expression of inflammatory markers, such as tumor necrosis factor (TNF)- $\alpha$ and interleukin (IL)-6, induced by LPS, and inhibited the phosphorylation of mitogen-activated protein kinases (MAPKs) and signal transducer and activator of transcription (STAT)1/STAT3. Resveratrol also upregulated the production of suppressor of cytokine signaling 1 (SOCS1; a STAT inhibitor) and suppressed the expression of miR-155, which plays an essential role in the innate and adaptive immune response. Given the elevated levels of SOCS1 in LPS-induced inflammation, our results suggest that resveratrol exerts antiinflammatory effects due to the upregulation of SOCS1, which is a potential target of miR-155, as well as of miR-155 mimics and inhibitors. These findings suggest the benefits of resveratrol, which are derived from its regulation of SOCS1 expression via the inhibition of miR-155, and indicate that resveratrol may be developed as a useful agent for the treatment of inflammatory diseases.
\end{abstract}

\section{Introduction}

Inflammation is an innate immune response mediated by macrophages and a panel of pro-inflammatory mediators, such as tumor necrosis factor $\alpha$ (TNF- $\alpha)$, interleukin (IL)-1 $\beta$, IL-6, nitric oxide (NO) and macrophage chemoattractant protein (MCP)-1. Macrophages promote inflammation and play a crucial role in cytokine secretion (1). Inflammatory diseases,

Correspondence to: Dr Wanru Cai, The Second Affiliated Hospital of Zhejiang Chinese Medicine University, 318 Chaowang Road, Hangzhou, Zhejiang 310005, P.R. China

E-mail: sundaymore@126.com

Key words: resveratrol, inflammation, cytokines, microRNA, Polygonum cuspidatum such as atherosclerosis (2), acute lung injury (3) and pulmonary fibrosis (4) are characterized by the overexpression of these cytokines and pro-inflammatory mediators. Thus, blocking the release of cytokines from activated macrophages may provide a mechanism for the treatment of inflammatory disorders.

Bacteria, viruses and alcohol promote the release of inflammatory cytokines from macrophages. Lipopolysaccharides (LPS) in the outer wall of Gram-negative bacteria are bound by Toll-like receptor 4 (TLR4) on the macrophage surface, thereby activating macrophages $(5,6)$ and triggering the activation of several intracellular signaling pathways, such as nuclear factor $\kappa \mathrm{B}(\mathrm{NF}-\kappa \mathrm{B})$, Janus kinase-signal transducers and activators of transcription (JAK-STATs) and mitogenactivated protein kinases (MAPKs). These signaling cascades regulate the expression of target genes involved in inflammatory cytokine production $(7,8)$.

Small endogenous RNA molecules known as microRNAs (miRNAs or miRs) have been identified as regulators of the inflammatory response, which act by specifically binding the 3'UTR of target miRNAs, marking them for degradation or suppressing translation (9). Several miRNAs have been implicated in the control of inflammatory processes, including miR-155, which plays a pro-inflammatory role in the LPS-stimulated immune response. The miRNA targets of miR155 include pro-apoptotic and anti-inflammatory proteins, such as the suppressor of cytokine signaling 1 (SOCS1) (10). SOCS1 inhibits JAK and STAT. It thereby creates a negative feedback loop in LPS-induced signaling pathways $(10,11)$.

Resveratrol is a polyphenolic compound found in grapes and traditional Chinese medicinal plants, such as Polygonum cuspidatum. It influences a variety of molecular targets, and many of them are associated with inflammation and immunity $(12,13)$. In this study, we examined the specific effects of resveratrol on the production of pro-inflammatory cytokines by LPS-stimulated RAW264.7 murine macrophages. Our findings suggest that resveratrol inhibits STAT activation and enhances SOCS1 expression by attenuating the production of miR-155.

\section{Materials and methods}

Materials and reagents. Resveratrol (>99\%, HPLC; molecular weight, 228.24, Trans-; Sigma-Aldrich, St. Louis, MO, USA) was dissolved in DMSO to produce an $80 \mathrm{mM}$ stock solution and stored at $-20^{\circ} \mathrm{C}$. The stock solution was diluted with medium to the desired concentration immediately 
prior to use. The SB203580 (Cat. no. S8307) and AG490 (Cat. no. T3434) were purchased from Sigma-Aldrich. Enzyme-linked immunosorbent assay (ELISA) kits for murine IL- 6 and TNF- $\alpha$, sICAM1 and CXCL10 were obtained from R\&D Systems (Minneapolis, MN, USA). The BCA ${ }^{\mathrm{TM}}$ protein assay kit and MTT reagent were purchased from Beyotime (Shanghai, China). Escherichia coli LPS (O55:B5) was obtained from Sigma-Aldrich. Antibodies directed against phosphorylated (p-)p38 MAPK (Thr180/Tyr182; Cat. no. 9211), p44/42 MAPK [extracellular signal-regulated kinase1/2 (ERK1/2); Cat. no. 4695],p-p44/42 MAPK (ERK1/2; Cat. no. 4376), STAT1 (42H3; Cat. no. 9175S), p-Tyr701 STAT1 (p-STAT1; Cat. no. 7649S), STAT3 (79D7; Cat. no. 4904), p-Tyr705 STAT3 (pSTAT3; Cat. no. 9145), SOCS1 (Cat. no. 3950) and $\beta$-actin (Cat. no. 4970), and HRP-conjugated anti-rabbit IgG (Cat. no. 7074) were obtained from Cell Signaling Technology (Beverly, MA, USA). Antibodies against c-Jun NH2-terminal kinase (JNK)1 (Cat. no. 3496-1), JNK1 (pY185)/JNK2(pY185)/JNK3(Py223) (Cat. no. 2155-1) and Crk/p38 (Cat. no. 5359-1) were purchased from Epitomics (Burlingame, CA, USA). The mimic and inhibitor of miR-155 (micrON ${ }^{\mathrm{TM}}$ mmu-miR-155-5p mimic, micrOFF ${ }^{\mathrm{TM}}$ mmu-miR-155-5p inhibitor, micrON ${ }^{\mathrm{TM}}$ mimic and micrOFF ${ }^{\mathrm{TM}}$ inhibitor negative control) and the Bulge-loop ${ }^{\mathrm{TM}}$ miRNA RT-qPCR primers for miR-155 and U6 (internal control for normalization) were purchased from Guangzhou RiboBio Co., Ltd. (Guangzhou, China). Dulbecco's modified Eagle's medium (DMEM), fetal bovine serum (FBS), and TRIzol reagent were purchased from Gibco/BRL (Grand Island, NY, USA).

Cell culture. The RAW264.7 murine macrophages were purchased from the American Type Culture Collection (ATCC, Rockville, MD, USA). The cells were cultivated in DMEM supplemented with $10 \%$ heat-inactivated FBS and antibiotics (100 U/ml penicillin and $100 \mu \mathrm{g} / \mathrm{ml}$ streptomycin) at $37^{\circ} \mathrm{C}$ in an atmosphere containing $5 \% \mathrm{CO}_{2}$.

Cell viability assay. For cell viability assays, the cells $\left(1 \times 10^{4}\right.$ cells/well) were seeded in 96-well plates, pre-treated with $1,5,10,20$, or $40 \mu \mathrm{M}$ resveratrol for $1 \mathrm{~h}$, and then stimulated with $1 \mu \mathrm{g} / \mathrm{ml}$ LPS for $24 \mathrm{~h}$. Cell viability was assessed by MTT assay according to the manufacturer's instructions. The results are expressed as fold changes relative to the control. Three replicates were performed for each treatment.

Determination of cytokine secretion. The cells were seeded in 24-well culture plates at a density of $1 \times 10^{5}$ cells/well and serum-starved overnight prior to treatment. Following adhesion, the cells were pre-incubated with resveratrol $(1,5,10$, and $20 \mu \mathrm{M}$ ) for $1 \mathrm{~h}$ and stimulated with $1 \mu \mathrm{g} / \mathrm{ml}$ LPS for $24 \mathrm{~h}$. The culture media were collected and centrifuged at $1,000 \mathrm{x}$ g to remove debris. IL-6, TNF- $\alpha$, soluble intercellular adhesion molecule 1 (sICAM1) and C-X-C motif chemokine 10 (CXCL10) in the media were quantified by ELISA according to manufacturer's instructions. The absorbance was read at $450 \mathrm{~nm}$ using a microplate reader (Bio-Rad Laboratories Inc., Hercules, CA, USA), and cytokine levels were calculated from standard curves. Three replicates were performed for each treatment.
Determination of cytokine expression. The cells were seeded in 6-wells culture plates at a density of $1 \times 10^{6}$ cells/well and serum-starved overnight. The cells were then pre-incubated with resveratrol $(1,5,10$, and $20 \mu \mathrm{M})$ for $1 \mathrm{~h}$ and stimulated with $1 \mu \mathrm{g} / \mathrm{ml}$ LPS for $4 \mathrm{~h}$. Total RNA was isolated using TRIzol reagent according to manufacturer's instructions, and as previously described (14) and single-strand cDNA was synthesized from $2 \mu \mathrm{g}$ total RNA using the PrimeScript ${ }^{\mathrm{TM}}$ II 1st-strand cDNA Synthesis kit (Takara Biotechnology,Co.,Ltd., Dalian, China). qPCR was performed on a C1000 Thermal Cycler (Bio-Rad) with SYBR-Green (Invitrogen, Carlsbad, CA, USA). Each $25 \mu 1$ reaction contained $12.5 \mu 1$ SYBR Premix, $0.5 \mu \mathrm{l}$ each primer $(10 \mu \mathrm{M}), 1 \mu \mathrm{l}$ cDNA and $10.5 \mu \mathrm{l}$ RNase-free $\mathrm{dH}_{2} \mathrm{O}$. The cycling conditions were as follows: step $1,94^{\circ} \mathrm{C}$ for $3 \mathrm{~min}$; step 2,35 cycles at $94^{\circ} \mathrm{C}$ for $20 \mathrm{~s}, 57^{\circ} \mathrm{C}$ for $20 \mathrm{sec}, 72^{\circ} \mathrm{C}$ for $30 \mathrm{sec}$; step 3, dissociation. The data were collected and analyzed using on-instrument software. Relative gene expression was determined by the $2^{-\Delta \Delta \mathrm{Ct}}$ method, as previously described (15). The primer sequences were as follows: TNF- $\alpha$ sense, 5'-GCAGAGAGGTTGACTTTC-3' and antisense, 5'-CTACTCCCAGGTTCTCTTCAA-3'; IL-6 sense, 5'-AGTTGTGCAATGGCAATTCTGA-3' and antisense, 5'-AGGACTCTGGCTTTGTCTTTCT-3'; sICAM sense, 5'-AGAAGGACTGCTTGGGGAA-3' and antisense, 5'-CCT CTGGCGGTAATAGGTG-3'; CXCL10 sense, 5'-GGATCCC TCTCGCAAGGA-3' and antisense, 5'-ATCGTGGCAA TGATCTCAACA-3'; SOCS1 sense, 5'-CACTTCTGGCTGG AGACC-3' and antisense, 5'-TGGAGAGGTAGGAGT GGAA-3'; and $\beta$-actin sense, 5'-TGCTGTCCCTGTA TGCCTCT-3' and antisense, 5'-TTTGATGTCACGCACG ATTT-3'. Each assay was normalized to $\beta$-actin.

Analysis of miR-155 expression by qPCR. Total cellular RNA was obtained as described above. The reverse transcription (RT) of $1 \mu \mathrm{g}$ total RNA was performed using the PrimeScript ${ }^{\mathrm{TM}}$ RT reagent kit (Takara Biotechnology, Co., Ltd.). Stem-loop RT-PCR was performed with SYBR-Green (Invitrogen). The cycling conditions were as follows: step $1,94^{\circ} \mathrm{C}$ for $3 \mathrm{~min}$; step 2, 35 cycles at $94^{\circ} \mathrm{C}$ for $15 \mathrm{sec}, 57^{\circ} \mathrm{C}$ for $15 \mathrm{sec}, 72^{\circ} \mathrm{C}$ for $25 \mathrm{sec}$; step 3, dissociation. Each assay was performed in triplicate and normalized to U6 expression. The primer sequences for miR155 and U6 are the property of Guangzhou RiboBio Co., Ltd.

Protein extraction and western blot analysis. The cells were seeded in 6-well culture plates at a density of $1 \times 10^{6}$ cells/well and serum-starved overnight. The cells were pre-incubated with resveratrol $(1,5,10$, and $20 \mu \mathrm{M})$ for $1 \mathrm{~h}$ and then stimulated with $1 \mu \mathrm{g} / \mathrm{ml}$ LPS for $30 \mathrm{~min}$ prior to assaying for p-p38 MAPK, p-AKT, p-p44/42 MAPK and p-JNK; and for $2 \mathrm{~h}$ prior to assaying for p-STAT1 and p-STAT3; LPS stimulation was performed for $24 \mathrm{~h}$ for SOCS1 analysis. We used SB203580 $(20 \mu \mathrm{M})$ and AG490 $(20 \mu \mathrm{M})$ to specifically block p38 MAPK and JAK, respectively. The RAW264.7 cells were pre-treated with resveratrol, SB203580 or AG490 for $1 \mathrm{~h}$, followed by stimulation with LPS. Cell lysates were obtained at $30 \mathrm{~min}$ and $2 \mathrm{~h}$ following the LPS challenge and the levels of p-p38 and p-STAT1/STAT3 were assessed by western blot analysis.

Following incubation, the cells were harvested and washed 3 times with ice-cold PBS. Total cellular protein was extracted with cell lysis buffer (Cell Signaling Technology, Beverly, MA, 

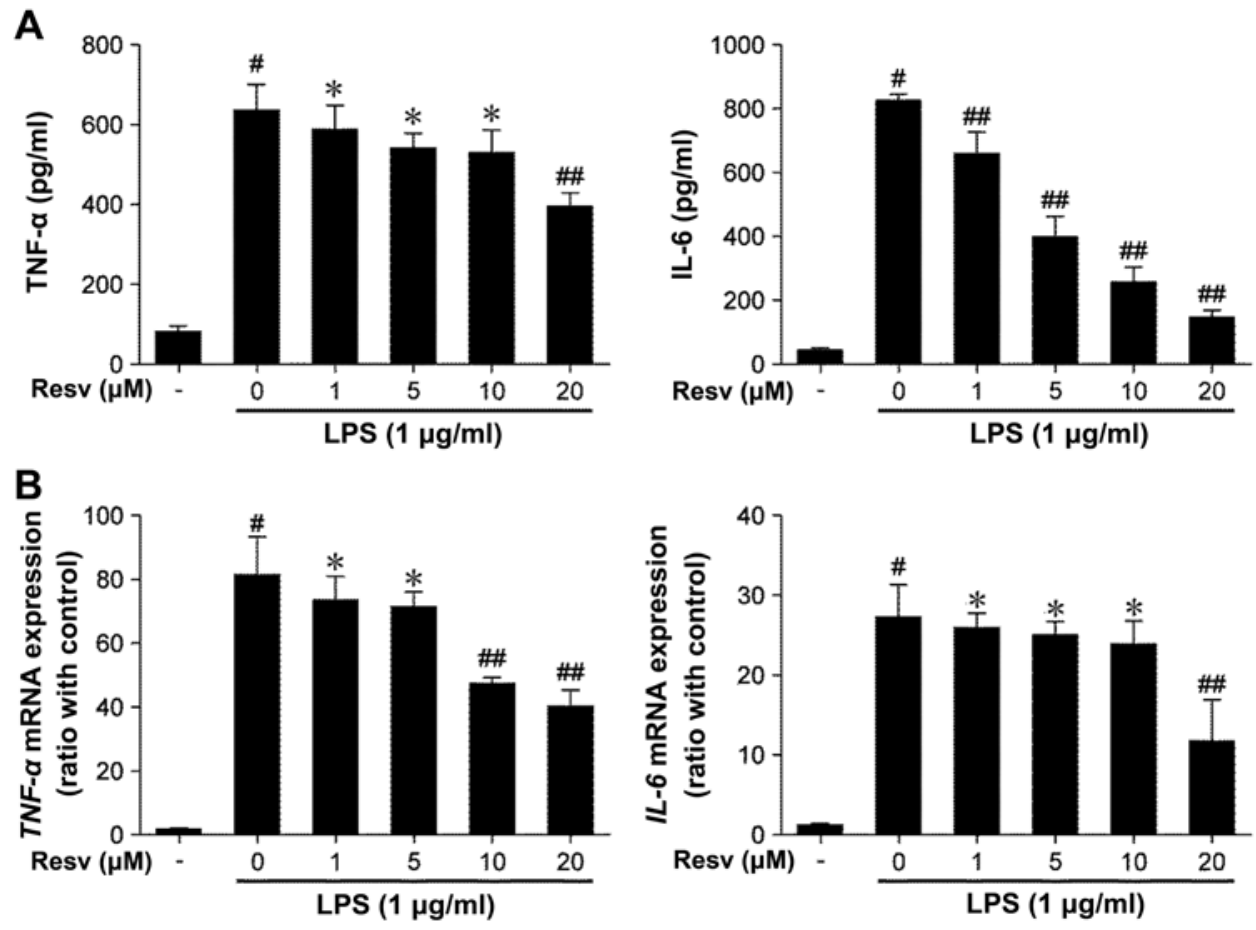

Figure 1. Resveratrol reduces tumor necrosis factor $\alpha$ (TNF- $\alpha$ ) and interleukin (IL)-6 production. RAW264.7 cells were treated with various concentrations resveratrol in the presence or absence of $1 \mu \mathrm{g} / \mathrm{ml}$ lipopolysaccharide (LPS) for $24 \mathrm{~h}$ prior to the protein assays and $4 \mathrm{~h}$ for microRNA (miRNA) expression assays. (A) TNF- $\alpha$ and IL-6 in the culture media were measured by enzyme-linked immunosorbent assay (ELISA). (B) TNF- $\alpha$ and IL- 6 transcript levels were determined by RT-qPCR vs. $\beta$-actin as the endogenous control. Data are presented as ratios against a blank control. The results are the means \pm SD of 3 independent experiments. ${ }^{\#} \mathrm{P}<0.01$ vs. blank control, ${ }^{*} \mathrm{P}<0.05$ vs. LPS group, ${ }^{\# \#} \mathrm{P}<0.01$ vs. LPS group. Blank control, untreated cells; LPS group, cells stimulated with only LPS.

USA) and quantified using a bicinchoninic acid protein assay kit (Beyotime). Equal amounts of lysate (30-50 $\mu$ g protein) were separated by SDS-PAGE and transferred onto nitrocellulose membranes (Millipore, Billerica, MA, USA). The membranes were blocked with 5\% BSA-Tris-buffered saline with Tween-20 for $1 \mathrm{~h}$ and incubated overnight at $4^{\circ} \mathrm{C}$ with primary monoclonal antibodies. The membranes were incubated with HRP-conjugated anti-rabbit $\mathrm{IgG}$ for $2 \mathrm{~h}$ at room temperature after washing 3 times in TBST, and the bands were visualized with a chemiluminescent substrate (ECL-Plus; Millipore) for 2-5 min using Quantity One (v. 4.62) software (Bio-Rad Laboratories Inc.).

Small interfering RNA (siRNA) and miRNA transfection. siRNA sequences targeting SOCS1 were designed by Guangzhou RiboBio Co., Ltd.. The siRNA was transfected into the RAW264.7 cells according to the manufacturer's instructions using Lipofectamine ${ }^{\circledR}$ RNAiMAX (Invitrogen). The cells were incubated with $10 \mathrm{nM}$ SOCS1 siRNA for $6 \mathrm{~h}$. Following transfection, the supernatant was replaced with fresh medium and the cells were pre-treated with resveratrol, followed by stimulation with LPS.

The mmu-miR-155-5p inhibitor and negative control (10 nM) were transfected into the cells for $6 \mathrm{~h}$; the cells were washed and pre-treated with resveratrol, followed by stimulation with LPS.

Statistical analysis. All data are expressed as the means \pm SEM. For statistical comparisons, the data were analyzed by ANOVA and Scheffe's post-hoc test or the Kruskal-Wallis and Mann-Whitney test. A P-value $<0.05$ was considered to indicate a statistically significant difference.

\section{Results}

Effect of resveratrol on cellular cytotoxicity. The cytotoxicity of resveratrol to RAW264.7 murine macrophages was determined by MTT assay. Resveratrol had no effect on cell viability following treatment for $24 \mathrm{~h}$ at concentrations of $0-20 \mu \mathrm{M}$ (data not shown), indicating no cytotoxic effects at the dosages and time points used in this study. For all subsequent experiments, non-toxic concentrations of resveratrol $(0-20 \mu \mathrm{M})$ were used.

Resveratrol suppresses the production of cytokines in LPS-stimulated RAW264.7 cells. LPS induces the release of cytokines from macrophages (16). Thus, in this study, we investigated whether resveratrol inhibits the production of cytokines in LPS-stimulated RAW264.7 cells. Stimulation with LPS alone induced the production of TNF- $\alpha$ and IL-6, whereas treatmetn with resveratrol inhibited the release of these cytokines in a dose-dependent manner (Fig. 1A).

We then investigated whether resveratrol influences the miRNA expression of TNF- $\alpha$ and IL-6. Indeed, resveratrol downregulated the miRNA expression of these cytokines (Fig. 1B). These results suggest that resveratrol attenuates the transcript and protein expression of inflammatory cytokines in LPS-stimulated RAW264.7 macrophages.

Resveratrol inhibits the LPS-induced activation of p38 MAPK and JAK/STATs. MAPKs are the mediators of important signaling events that control the synthesis and release of inflammatory cytokines by activated macrophages (17). Thus, in this study, to determine whether resveratrol influences the LPS-mediated activation of MAPK signaling, we measured 
A
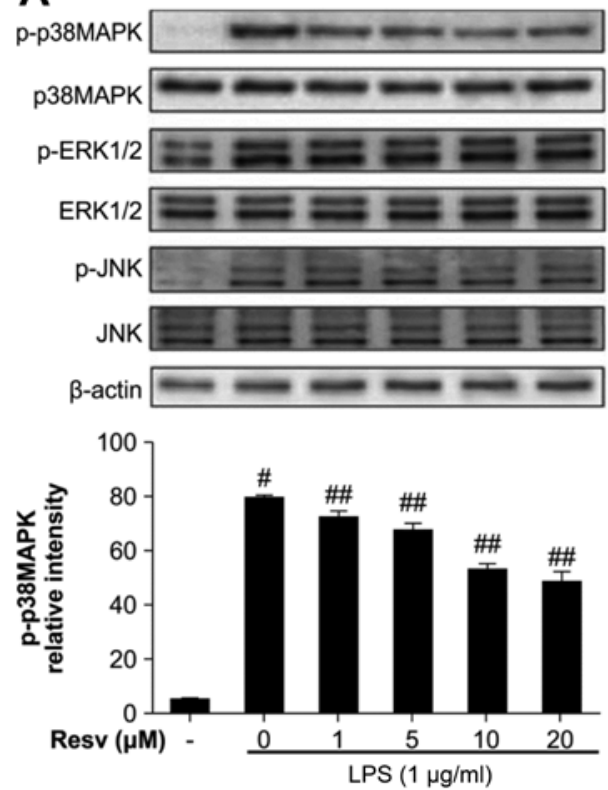

B
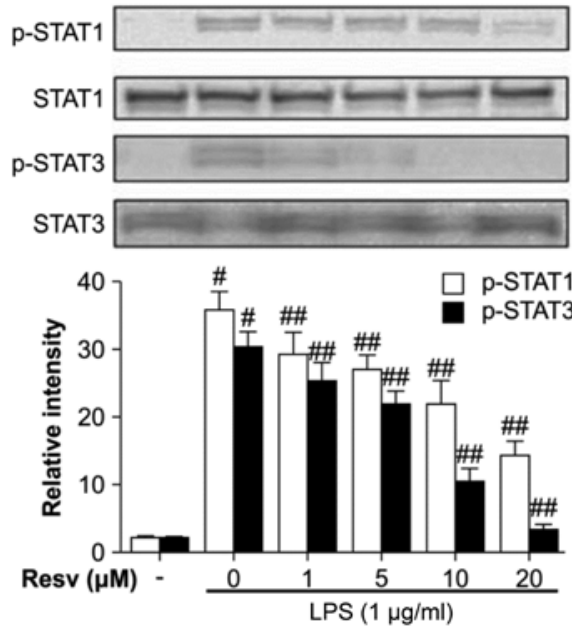

C

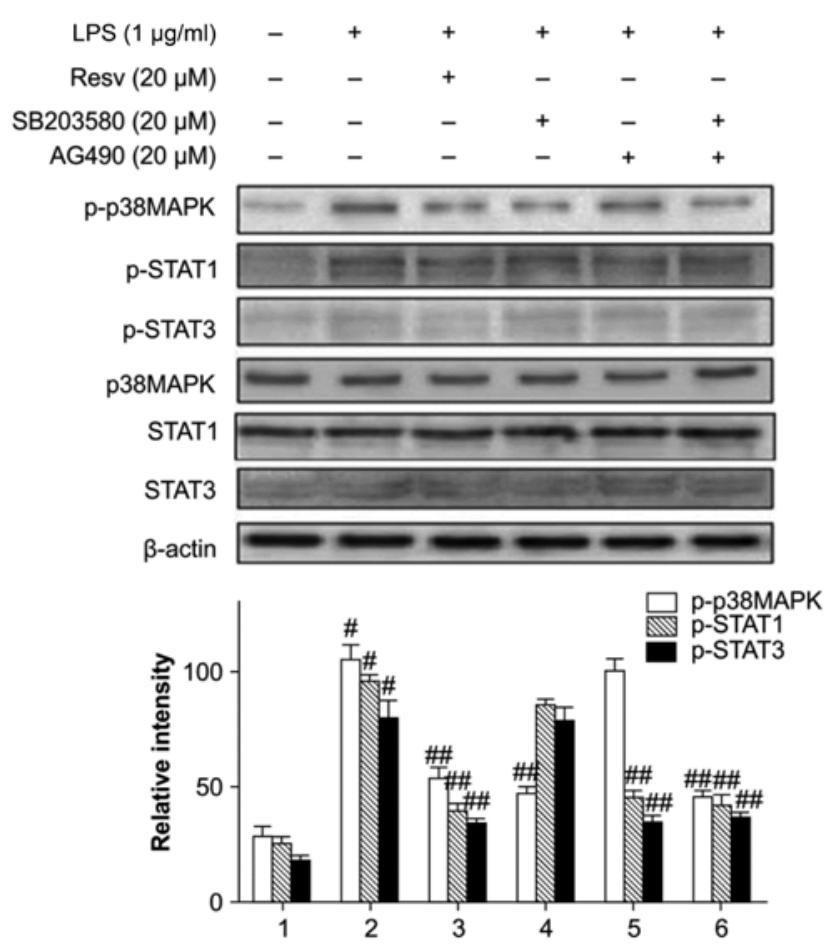

Figure 2. Resveratrol reduces lipopolysaccharide (LPS)-induced mitogen-activated protein kinase (MAPK) and signal transducer and activator of transcription (STAT) activity. Cells were pre-treated with resveratrol for $1 \mathrm{~h}$ and then stimulated with $1 \mu \mathrm{g} / \mathrm{ml} \mathrm{LPS}$ for (A) $30 \mathrm{~min}$ or (B) $2 \mathrm{~h}$. (C) The cells were pre-treated with $20 \mu \mathrm{M}$ resveratrol, SB20350, or AG490 for $1 \mathrm{~h}$ and then stimulated with LPS for $30 \mathrm{~min}$ and $2 \mathrm{~h}$. Western blot analysis was performed with the indicated antibodies. Bands were quantified by densitometry and the results are presented as the means \pm SD of 3 independent experiments. ${ }^{\#} \mathrm{P}<0.01$ vs. blank control; ${ }^{\# \prime} \mathrm{P}<0.01$ vs. LPS group. Blank control, untreated cells; LPS group, cells stimulated with only LPS.

the active form of MAPK signaling components using specific antibodies to p-ERK1/2, p-JNK1/2 and p-p38 MAPK. LPS rapidly activated the phosphorylation of p38, ERK1/2, JNK1/2 (all $\mathrm{P}<0.01)$. Resveratrol inhibited the phosphorylation of p38 in a dose-dependent manner, but had no effect on ERK1/2 and JNK1/2 phosphorylation (Fig. 2A).

STAT1 and STAT3 are key transcription factors in immunity and play roles in the inflammatory signaling cascades triggered by LPS (18). In this study, we examined the hypothesis that resveratrol blocks the LPS-induced phosphorylation of
STAT1 and STAT3. Indeed, resveratrol inhibited LPS-induced STAT1 and STAT3 phosphorylation in RAW264.7 cells in a dose-dependent manner (Fig. 2B).

We then compared resveratrol to other signaling inhibitors from the LPS-induced inflammatory response. We used SB203580 and AG490 to specifically block p38 MAPK and JAK, respectively. SB203580 only inhibited p38 phosphorylation and AG490 inhibited STAT1/STAT3 phosphorylation. By contrast, resveratrol inhibited both factors and this indicates that it has a broader range of inhibitory activity (Fig. 2C). 
A

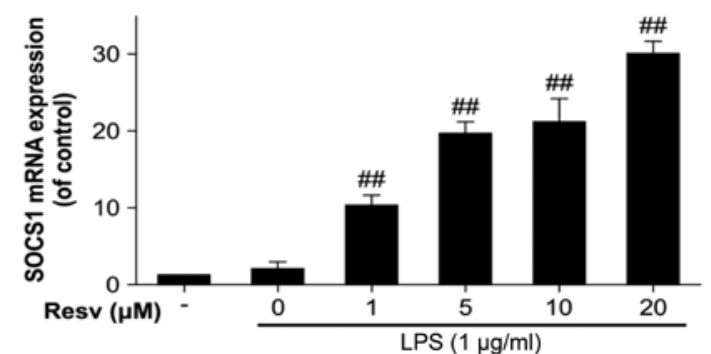

B
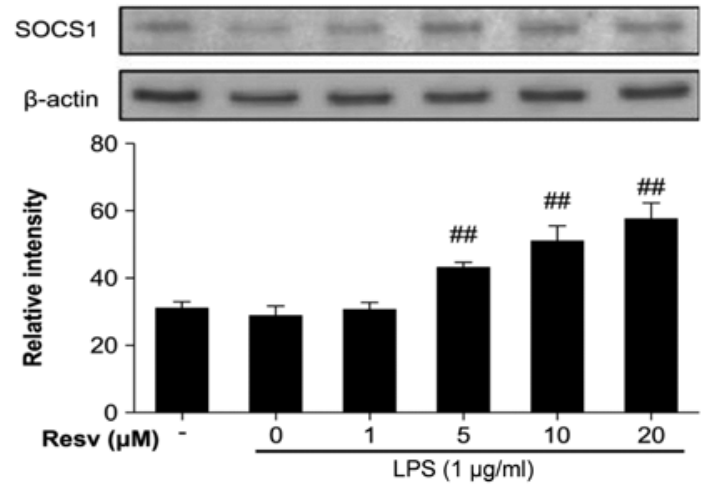

C

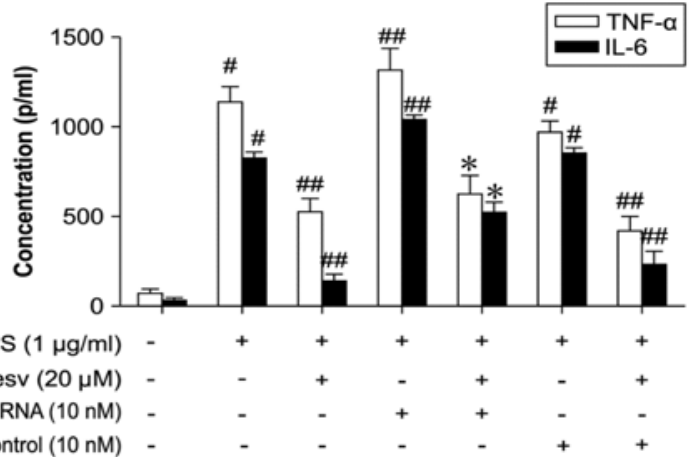

Figure 3. Effect of resveratrol on suppressor of cytokine signaling 1 (SOCS1) production in lipopolysaccharide (LPS)-induced inflammation. Cells were pre-treated with resveratrol for $1 \mathrm{~h}$ and then stimulated with LPS for (A) $4 \mathrm{~h}$ for microRNA (miRNA) and (B) $24 \mathrm{~h}$ for protein expression. (C) Cells were transfected with SOCS1-small interfering RNA (siRNA) for $6 \mathrm{~h}$, then treated with resveratrol for $1 \mathrm{~h}$ and stimulated with LPS for $24 \mathrm{~h}$; tumor necrosis factor $\alpha$ (TNF- $\alpha$ ) and interleukin (IL)- 6 were measured by enzyme-linked immunosorbent assay (ELISA). ${ }^{\text {} \mathrm{P}}<0.01$ vs. blank control, ${ }^{\# \#} \mathrm{P}<0.01$ vs. LPS group, ${ }^{*} \mathrm{P}<0.01$ vs. LPS + Resv group ir LPS + SOCS1-siRNA group. Blank control, untreated cells; LPS group, cells stimulated with only LPS; LPS + Resv group, cells were treated with resveratrol then stimulated with LPS; LPS + SOCS1siRNA, cells were transfected with SOCS1-siRNA then stimulated with LPS.

Resveratrol upregulates SOCS1 expression in LPS-stimulated RAW264.7 cells. SOCS proteins function via the suppression of the JAK/STAT pathway. SOCS1 acts as a pseudo-substrate, interacting with and inhibiting JAK tyrosine kinase activity, thereby suppressing cytokine signal transduction (19). In this study, we examined whether resveratrol induces SOCS1 expression to suppress JAK/STAT signaling in LPS-stimulated RAW264.7 cells pre-treated with resveratrol. Indeed, resveratrol induced SOCS1 expression particularly at a higher concentration, indicating its function as an anti-inflammatory agent via the promotion of SOCS1 expression (Fig. 3A and B).

We then used siRNA targeting SOCS1 to confirm the status of SOCS1 in this inflammatory response. Following transfection of the cells with SOCS1 siRNA, TNF- $\alpha$ and IL-6 expression increased in the LPS-stimulated macrophages and the anti-inflammatory effect of resveratrol was somewhat reduced (Fig. 3C).

Resveratrol downregulates miR-155 in LPS-stimulated $R A W 264.7$ cells. miR-155 plays an important role in modulating immune processes (20). Thus, we determined whether resveratrol influences miR-155 production by measuring miR-155 expression with a stem-loop RT-PCR method. LPS alone induced miR-155 expression, whereas resveratrol inhibited its expression in a dose-dependent manner (Fig. 4A).

We used micrOFF ${ }^{\mathrm{TM}}$ mmu-miR-155-5p inhibitor $(10 \mathrm{nM})$ to neutralize miR-155 expression and the micrON ${ }^{\mathrm{TM}}$ mmu-miR-155-5p mimic ( $5 \mathrm{nM}$ ) in resveratrol- and LPS-treated cells and observed an increased expression of SOCS1 in the presence of miR-155-5p inhibition and a decreased expression with miR-155-5p overexpression (Fig. 4B-D). These results demonstrated that resveratrol upregulated SOCS1 by downregulating miR-155 and has the same effect as an miR-155 inhibitor.

\section{Discussion}

Resveratrol is a polyphenolic compound found in grapes and in the Chinese herb, Polygonum cuspidatum. It interacts with multiple molecular targets, many of them associated with inflammation and immunity (13). In this study, we provide strong evidence that resveratrol suppresses the production of pro-inflammatory cytokines and inhibits the activation of the p38 MAPK and STAT1/STAT3 signaling pathways by upregulating SOCS1 expression in response to LPS stimulation.

LPS, the major component of the cell wall of Gram-negative bacteria, interacts with TLR4 on macrophages, which then produce pro-inflammatory cytokines, such as IL- 6 , TNF- $\alpha$, CXCL10. These pro-inflammatory cytokines mediate cell damage and tissue destruction $(21,22)$. TNF- $\alpha$ is the earliest and most important cytokine during the inflammatory reaction, which can activate macrophages and then promote the release of various mediators $(23,24)$. Several important common pathways have been identified, including the MAPK pathways. The MAPKs are intracellular serine/threonine protein kinases and include ERK1/2, p38 MAPK and JNK. They are involved in diverse cellular processes, including cell growth, proliferation, differentiation, cell death and immune responses (25). In our study, resveratrol inhibited the expression of IL- 6 and TNF- $\alpha$ at the miRNA and protein level. Resveratrol has been shown to modulate the LPS-TLR4 pathway and suppress the activation of nuclear factor $(\mathrm{NF})-\mathrm{\kappa B}(26,27)$. Furthermore, in our study, resveratrol inhibited the LPS-induced phosphorylation of p-38 MAPK, but not of that ERK1/2 and JNK, indicating that $\mathrm{p}-38$ MAPK is a molecular target for resveratrol. By using specific kinase inhibitors for p-38 MAPK, we confirmed this anti-inflammatory effect.

The JAK-STAT cascade is an essential signaling pathway in the immune and inflammatory responses (28). LPS receptor binding induces the phosphorylation of receptor-associated JAK, which in turn leads to STAT phosphorylation. In addition to LPS, other stimuli such as cytokines and growth factors can also activate JAK-STAT signaling systems (29). Phosphorylated STATs are dissociated from the receptor complex and then form homodimers or heterodimers, which translocate to the nucleus 
A
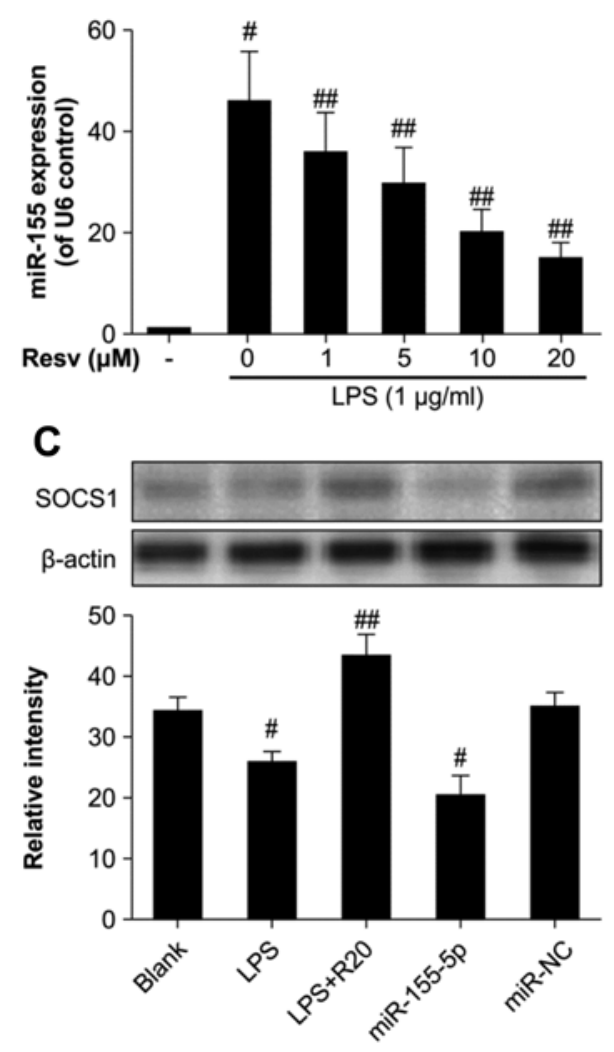

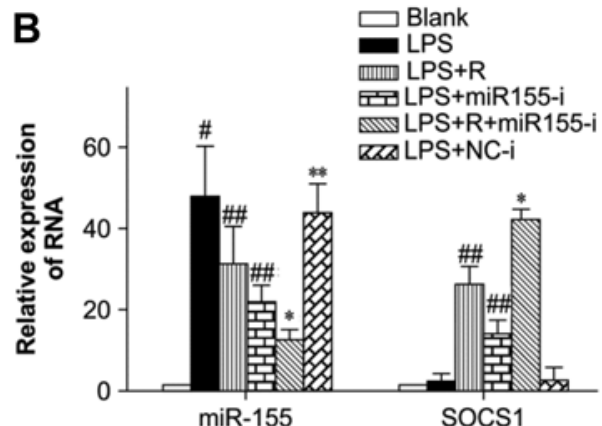

D
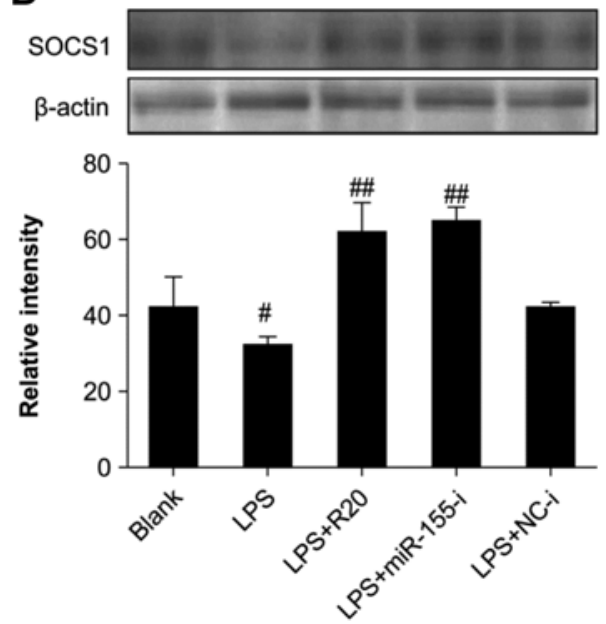

Figure 4. The influence of resveratrol on microRNA (miR)-155 expression in lipopolysaccharide (LPS)-induced inflammation. (A) Cells were pre-treated with resveratrol for $1 \mathrm{~h}$ and then stimulated with $1 \mu \mathrm{g} / \mathrm{ml}$ LPS for $4 \mathrm{~h}$. miR-155 expression was assayed by stem-loop RT-PCR with U6 as an endogenous control. (B) Cells were transfected with miR-155 inhibitor and miRNA negative control, then treated with $20 \mu \mathrm{M}$ resveratrol and $1 \mu \mathrm{g} / \mathrm{ml} \mathrm{LPS}$ as above, suppressor of cytokine signaling 1 (SOCS1) expression was normalized against the $\beta$-actin control. (C and D) Cells were transfected with miR-155 mimic and inhibitor, then treated as above and SOCS1 was assayed by western blot analysis. Bands were quantified by densitometry. The results are presented as the means \pm SD of 3 independent experiments. ${ }^{\#} \mathrm{P}<0.01$ vs. blank control, ${ }^{\# \#} \mathrm{P}<0.01$ vs. LPS group, ${ }^{*} \mathrm{P}<0.01$ vs. LPS + Resv group and LPS + miR155-i group, ${ }^{* *} \mathrm{P}<0.01$ vs. blank control but P $>0.05$ vs. LPS group. Blank control, untreated cells; LPS group, cells stimulated with only LPS; LPS + R20, cells stimulated with LPS and treated with resveratrol at $20 \mu \mathrm{M}$; LPS + miR155-i group, cells transfected with miR-155 inhibitor then stimulated with LPS.

where they regulate the transcription of pro-inflammatory target genes. STAT1 and STAT3 are vital modulators in inflammatory signaling cascades triggered by LPS (30). In this study, resveratrol suppressed the phosphorylation of STAT1 and STAT3 $2 \mathrm{~h}$ after the LPS challenge. We noted STAT1 and STAT3 phosphorylation was decreased much later than the activation of MAPK, indicating that STAT1/3 may be downstream targets of resveratrol. Previous studies have suggested that the serine 727 of STAT1 and STAT3 can be phosphorylated by p38 MAPK (31-34). However, we did not find evidence indicating that the phosphorylation of STAT1 and STAT3 is induced by p38 MAPK, as SB203580, a specific inhibitor of p38 MAPK, did not block the downstream signal of STAT1/3. We suggest that resveratrol may have extensive anti-inflammatory effects as it interferes with p38 MAPK and STAT1/STAT3.

SOCS1 plays a vital role in the negative regulation of cytokines and TLR-mediated signaling pathways (35). SOCS1 blocks signaling by interacting with phosphotyrosine residues of JAK2 and STATs (19). SOCS1-deficient macrophages secrete more pro-inflammatory cytokines such as TNF- $\alpha$ and IL-6 (36). In our study, SOCS1 transcript expression was slightly upregulated in LPS-stimulated macrophages; however, resveratrol increased SOCS1 expression, particularly at higher concentrations. We speculated that resveratrol acts as an anti-inflammatory agent partly due to the upregulation of SOCS1 expression, and SOCS1 negatively regulates inflammation. This effect is lost upon the RNA silencing of SOCS1 in RAW264.7 macrophages. Thus, the absence of SOCS1 caused more inflammatory cytokines to be released, such as TNF- $\alpha$ and IL-6 than the presence of SOCS1. We confirmed that resveratrol exerted an anti-inflammatory effect by enhancing SOCS1 expression.

miRNAs regulated immune responses. miR-155 has been found in several immune cell types, such as macrophages, monocytes and dendritic cells (37). Many of the miR-155 targets encode anti-inflammatory proteins, such as SOCS1. LPS can induce the expression of miR-155, and the effect of miR155 is to combine with the 3'UTR miRNA of SOCS1, then downregulate the protein expression of SOCS1. In this study, a decrease in SOCS1 expression was observed following transfection with a miR-155-5p mimic, which caused the overexpression of miR-155, and induced the expression of pro-inflammatory cytokines. By contrast, an increase in SOCS1 expression had the opposite effect following transfection with a miR-155 inhibitor, and had the same effect as resveratrol. Our study provides compelling evidence of the inhibition of miR-155 by resveratrol through the upregulation of SOCS1. The mutual restrictive 
associatoin between miR-155 and SOCS1 may be the mechanism responsible for the anti-inflammatory effect of resveratrol. SOCS1, as a negative regulator, inhibited the TLR-mediated JAK/STAT inflammatory cascade. Resveratrol also inhibited the p38 MAPK signaling pathway. Thus, resveratrol exerts multiple anti-inflammatory effects in LPS-stimulated inflammatory cells. This study highlights the potential therapeutic value of resveratrol in the treatment of inflammatory diseases.

\section{Acknowledgements}

This study was funded by National Natural Science Foundation of China (grant no. 81273678).

\section{References}

1. Brummer E, Capilla J, Bythadka L and Stevens DA: Production of IL-6, in contrast to other cytokines and chemokines, in macrophage innate immune responses: effect of serum and fungal (Blastomyces) challenge. Cytokine 39: 163-170, 2007.

2. Libby P: Inflammation in atherosclerosis. Nature 420: 868-874, 2002.

3. Goodman RB, Pugin J, Lee JS and Matthay MA: Cytokine-mediated inflammation in acute lung injury. Cytokine Growth Factor Rev 14: 523-535, 2003.

4. Driscoll KE, Maurer JK, Higgins J and Poynter J: Alveolar macrophage cytokine and growth factor production in a rat model of crocidolite-induced pulmonary inflammation and fibrosis. J Toxicol Environ Health 46: 155-169, 1995.

5. Berghaus LJ, Moore JN, Hurley DJ, Vandenplas ML, Fortes BP, Wolfert MA and Boons GJ: Innate immune responses of primary murine macrophage-lineage cells and RAW 264.7 cells to ligands of toll-like receptors 2,3, and 4. Comp Immunol Microbiol Infect Dis 33: 443-454, 2010.

6. Froidevaux C, Roger T, Martin C, Glauser MP and Calandra T: Macrophage migration inhibitory factor and innate immune responses to bacterial infections. Crit Care Med 29 (Suppl 7): S13-S15, 2001.

7. Roger T, Chanson AL, Knaup-Reymond M and Calandra T: Macrophage migration inhibitory factor promotes innate immune responses by suppressing glucocorticoid-induced expression of mitogen-activated protein kinase phosphatase-1. Eur J Immunol 35: 3405-3413, 2005.

8. Baeuerle PA: IkappaB-NF-kappaB structures: at the interface of inflammation control. Cell 95: 729-731, 1998

9. Juarez MT, Kui JS, Thomas J, Heller BA and Timmermans MC: microRNA-mediated repression of rolled leaf1 specifies maize leaf polarity. Nature 428: 84-88, 2004.

10. Cardoso AL, Guedes JR, Pereira de Almeida L and Pedroso de Lima MC: miR-155 modulates microglia-mediated immune response by down-regulating SOCS-1 and promoting cytokine and nitric oxide production. Immunology 135: 73-88, 2012.

11. Kimura A, Naka T, Muta T, Takeuchi O, Akira S, Kawase I and Kishimoto T: Suppressor of cytokine signaling-1 selectively inhibits LPS-induced IL-6 production by regulating JAK-STAT Proc Natl Acad Sci USA 102: 17089-17094, 2005.

12. Gambini J, López-Grueso R, Olaso-González G, Inglés M, Abdelazid K, El Alami M, Bonet-Costa V, Borrás C and Viña J: Resveratrol: distribution, properties and perspectives. Rev Esp Geriatr Gerontol 48: 79-88, 2013 (in Spanish).

13. Švajger U and Jeras M: Anti-inflammatory effects of resveratrol and its potential use in therapy of immune-mediated diseases. Int Rev Immunol 31: 202-222, 2012.

14. Simões AE, Pereira DM, Amaral JD, Nunes AF, Gomes SE, Rodrigues PM, Lo AC, D'Hooge R, Steer CJ, Thibodeau SN, et al: Efficient recovery of proteins from multiple source samples after TRIzol( $\left({ }^{\circledR}\right)$ or TRIzol( $\left({ }^{\circ}\right)$ LS RNA extraction and long-term storage. BMC Genomics 14: 181, 2013.

15. Ihsan A, Wang X, Liu Z, Wang Y, Huang X, Liu Y, Yu H, Zhang H, Li T, Yang $C$ and Yuan Z: Long-term mequindox treatment induced endocrine and reproductive toxicity via oxidative stress in male wistar rats. Toxicol Appl Pharmacol 252: 281-288, 2011.
16. Bølling AK, Samuelsen JT, Morisbak E, Ansteinsson V, Becher R, Dahl JE and Mathisen GH: Dental monomers inhibit LPS-induced cytokine release from the macrophage cell line RAW264.7. Toxicol Lett 216: 130-138, 2013.

17. Huang JL, Zhang YL, Wang CC, Zhou JR, Ma Q, Wang X, Shen XH and Jiang CL: Enhanced phosphorylation of MAPKs by NE promotes TNF- $\alpha$ production by macrophage through $\alpha$ adrenergic receptor. Inflammation 35: 527-534, 2012.

18. Kaplan MH: STAT signaling in inflammation. JAK-STAT 2: e24198, 2013

19. Croker BA, Kiu H and Nicholson SE: SOCS regulation of the JAK/ STAT signalling pathway. Semin Cell Dev Biol 19: 414-422, 2008

20. Vigorito E, Kohlhaas S, Lu D and Leyland R: miR-155: an ancient regulator of the immune system. Immunol Rev 253: 146-157, 2013.

21. Garzón E, Holzmuller P, Bras-Gonçalves R, Vincendeau P, Cuny G, Lemesre JL and Geiger A: The Trypanosoma bruce gambiense secretome impairs lipopolysaccharide-induced maturation, cytokine production, and allostimulatory capacity of dendritic cells. Infect Immun 81: 3300-3308, 2013

22. Chang FM, Reyna SM, Granados JC, Wei SJ, Innis-Whitehouse W, Maffi SK, Rodriguez E, Slaga TJ and Short JD: Inhibition of neddylation represses lipopolysaccharide-induced proinflammatory cytokine production in macrophage cells. J Biol Chem 287: 35756-35767, 2012.

23. Mukhopadhyay S, Hoidal JR and Mukherjee TK: Role of TNFalpha in pulmonary pathophysiology. Respir Res 7: 125, 2006.

24. Butt Y, Kurdowska A and Allen TC: Acute lung injury: A clinical and molecular review. Arch Pathol Lab Med 140: 345-350, 2016.

25. Trempolec N, Dave-Coll N and Nebreda AR: SnapShot: p38 MAPK signaling. Cell 152: 924, 2013.

26. Byun EB, Sung NY, Park JN, Yang MS, Park SH and Byun EH: Gamma-irradiated resveratrol negatively regulates LPS-induced MAPK and NF- $\mathrm{B}$ signaling through TLR4 in macrophages. Int Immunopharmacol 25: 249-259, 2015.

27. Ma C, Wang Y, Dong L, Li M and Cai W: Anti-inflammatory effect of resveratrol through the suppression of $\mathrm{NF}-\kappa \mathrm{B}$ and JAK/STAT signaling pathways. Acta Biochim Biophys Sin (Shanghai) 47: 207-213, 2015.

28. Mertens C and Darnell JE Jr: SnapShot: JAK-STAT signaling. Cell 131: 612, 2007.

29. O'Shea JJ, Gadina M and Schreiber RD: Cytokine signaling in 2002: new surprises in the Jak/Stat pathway. Cell 109 (Suppl): S121-S131, 2002.

30. Murray PJ: The JAK-STAT signaling pathway: Input and output integration. J Immunol 178: 2623-2629, 2007.

31. Kovarik P, Stoiber D, Eyers PA, Menghini R, Neininger A, Gaestel M, Cohen P and Decker T: Stress-induced phosphorylation of STAT1 at Ser727 requires p38 mitogen-activated protein kinase whereas IFN-gamma uses a different signaling pathway. Proc Natl Acad Sci USA 96: 13956-13961, 1999.

32. Shuai K and Liu B: Regulation of JAK-STAT signalling in the immune system. Nat Rev Immunol 3: 900-911, 2003.

33. Galdiero M, Vitiello M, D'Isanto M, Raieta K and Galdiero E: STAT1 and STAT3 phosphorylation by porins are independent of JAKs but are dependent on MAPK pathway and plays a role in U937 cells production of interleukin-6. Cytokine 36: 218-228, 2006.

34. Sakaguchi M, Oka M, Iwasaki T, Fukami Y and Nishigori C: Role and regulation of STAT3 phosphorylation at Ser727 in melanocytes and melanoma cells. J Invest Dermatol 132: 1877-1885, 2012

35. Linossi EM, Babon JJ, Hilton DJ and Nicholson SE: Suppression of cytokine signaling: the SOCS perspective. Cytokine Growth Factor Rev 24: 241-248, 2013.

36. Guenterberg KD, Lesinski GB, Mundy-Bosse BL, Karpa VI, Jaime-Ramirez AC, Wei L and Carson WE III: Enhanced anti-tumor activity of interferon-alpha in SOCS1-deficient mice is mediated by $\mathrm{CD}^{+}$and $\mathrm{CD}^{+} \mathrm{T}$ cells. Cancer Immunol Immunother 60: 1281-1288, 2011.

37. O'Connell RM, Taganov KD, Boldin MP, Cheng G and Baltimore D: MicroRNA-155 is induced during the macrophage inflammatory response. Proc Natl Acad Sci USA 104: 1604-1609, 2007. 